\title{
Ärztliche Weiterbildung: Staats- oder Standesaufgabe? Oder gar eine Aufgabe für Fakultäten?
}

\author{
U. M. Lütolf
}

Mit dem Personenfreizügigkeitsabkommen der EU musste die ärztliche Weiterbildung den «staatlichen Segen» erhalten. Dabei stellt sich die Frage nach den Rollen in der Ausgestaltung und Überwachung der ärztlichen Weiterbildung. Berufsorganisation, der Staat und die Fakultäten müssen eine geeignete Form des Zusammenwirkens finden, um die Qualität der Gesundheitsversorgung sicherzustellen.

Ein Blick nach Kanada, wo in langer Tradition Fakultäten, Standesorganisation und Staat zusammenarbeiten, kann hilfreich sein, auch bei uns einen geeigneten Weg für die ärztliche Weiterbildung zu finden.

Korrespondenz:

Prof. Dr. Urs Martin Lütolf

Klinikdirektor Radio-Onkologie

UniversitätsSpital

Rämistrasse 100

CH-8091 Zürich

Tel. 0442552930

Fax 0442554435

E-Mail: patricia.litschi@usz.ch

\section{Einleitung}

Im vergangenen Jahr wurde in der Schweiz erstmals die Akkreditierung der Facharzttitel durchgeführt. Das EDI hat per 1. Juni 2005 für alle Weiterbildungsprogramme der FMH die Akkreditierung ausgesprochen und dies auch den Präsidentinnen und Präsidenten der Fachgesellschaften mitgeteilt. Der Vorgang der Akkreditierung hat neben anderen die Frage aufgeworfen, wie weit eine Berufsorganisation wie die FMH eine solche Aufgabe ohne pekuniäre Eigeninteressen im Sinne der Wohlfahrt des Staates wahrnehmen kann. Seitens des EDI wird eine Lösung favorisiert, wonach verschiedene Organisationen gleichzeitig die ärztliche Weiterbildung reglementieren und der daraus resultierende Wettbewerb zu einer besseren und günstigeren Weiterbildung führen soll.

Dass sich der Bund mit der ärztlichen Weiterbildung befassen will, ist neu. Das Personenfreizügigkeitsabkommen mit der EU verlangt lediglich, dass alle in der EU-Richtlinie vorgesehenen Diplome und Facharzttitel staatlich anerkannt sind. Während sich die Ärzteschaft in Deutschland in den Landesärztekammern als öffentlichrechtliche Anstalten organisiert hat und in dieser Form politisch und juristisch unbestritten alle Weiterbildungsaufgaben wahrnimmt, musste in der Schweiz ein neuer Weg gefunden werden, da die bisherigen Facharzttitel der FMH keine offizielle staatliche Anerkennung genossen. Erst mit dem FMPG [1], das bis zur Inkraftsetzung des
MedBG [2] Gültigkeit hat, wurden die 43 Facharzttitel der FMH mittels Akkreditierung als eidgenössische Titel anerkannt.

Die Aufgabe des im FMPG geregelten eidgenössischen Weiterbildungsausschusses besteht u.a. darin, das EDI in Fragen Weiterbildung bei den akademischen Medizinalberufen zu beraten, $\mathrm{zu}$ den Akkreditierungsanträgen Stellung $\mathrm{zu}$ nehmen und den Trägerorganisationen (FMH, SSO) Massnahmen zur Erhöhung der Qualität der Weiterbildung vorzuschlagen.

Mit Blick auf mögliche Verbesserungen konnte ich mich mit der ärztlichen Weiterbildungsorganisation in Kanada befassen, dies aus folgendem Grund: Das staatliche kanadische Gesundheitswesen und die Ärzte in Kanada scheinen in langer Tradition einen Modus vivendi zur Realisierung der ärztlichen Weiterbildung gefunden zu haben. Die Aufgabenteilung könnte auch für uns von Interesse sein.

\section{Beobachtungen beim Royal College in Ottawa (Kanada): ein Blick hinter die ehemaligen Klostermauern}

Der Taxichauffeur am Busterminal von Ottowa kannte die Adresse bestens: Seine Frage, ob ich zu einer Prüfung gefahren werde müsse, konnte ich unbelastet mit Nein beantworten. Das ehemalige Nonnenkloster steht seit 1992 dem Royal College of Physicians and Surgeons of Canada als Hauptsitz zur Verfügung. Es ist äusserlich Zeichen einer Tradition, die sich im vollen Titel des Royal College spiegelt: Der Name «The Royal College of Physicians and Surgeons of Canada» könnte fast an die Zeiten erinnern, wo die Barbiere das Handwerk beherrschten und es daneben Ärzte gab. Gegründet wurde das Royal College of Physicians and Surgeons of Canada mit einem Parlamentsbeschluss 1929. Es ist eine private Non-Profit-Organisation, die 39000 medizinische Spezialisten einschliesst und von diesen auch finanziell getragen wird. Die Aufgabe («Mission») des College ist es, die höchsten Stan- 
dards und die Qualität der Gesundheitsversorgung sicherzustellen. Das Royal College will aber auch einen Beitrag zu einer gut fundierten Gesundheitspolitik leisten. Das College definiert die Standards und wacht über die Weiterbildung der medizinischen und chirurgischen «Spezialisten» (60 Spezialitäten oder Spezialprogramme). Auch die Regelung der kontinuierlichen Fortbildung ist dem Royal College übertragen. Die Titelträger ermöglichen durch ihre Jahresbeiträge das Funktionieren des Royal College, es fliessen keine staatlichen Gelder. Abgekoppelt von dieser fachlichen Weiterbildung ist die Lizenzierung (Berufsausübung), die den einzelnen Staaten in Kanada obliegt.

Geleitet wird das College von 24 Mitgliedern, je 12 aus Medizin und Chirurgie, die nach einem geographisch-linguistischen und nach Staaten verteilten Prinzip von der Basis her gewählt werden. Vier Mitglieder aus der Bevölkerung, weitere Fellows können ernannt werden. Ebenfalls sind die Weiterzubildenden im College vertreten. Jedes Jahr kommen 1600 Fellows aus dem ganzen Land freiwillig in verschiedenen Kommissionen zum Abnehmen von Examen und zum Ausarbeiten von Tests zusammen. 6500 Arbeitstage sollen so jedes Jahr geleistet werden.

Klar getrennt von dieser «Mission der Weiterund Fortbildung» beschäftigen sich in den einzelnen Staaten die dort organisierten Ärztegesellschaften mit Tarif- und Lohnfragen und nehmen so die finanziellen Interessen der Mitglieder wahr.

\section{Die Rolle des Staates?}

In Kanada wird politisch entschieden, wie viele Weiterbildungsstellen den staatlichen Spitälern zur Verfügung gestellt werden. Für die Spitäler sind diese Weiterbildungsstellen sehr erwünscht, und die Anstrengungen der entsprechenden Verantwortlichen für die Weiterbildung (Programmdirektoren), in den Fakultäten und Spitälern den Anforderungen des Royal College - das die entsprechenden Standards setzt - nachzukommen, sind sehr gross. Der Verlust der Weiterbildungsstellen wäre nicht nur ein Prestigeverlust, sondern auch ein Einschnitt im Bereich der Patientenversorgung und für die Kaderärzte (staff) der Spitäler eine Behinderung in ihren akademischen Aufgaben, da ihnen die entsprechenden Arbeitskräfte fehlen.

Die Organisation der Weiter- und Fortbildung wird von den medizinischen Fakultäten wahrgenommen, die in der Regel einen Pro- dekan mit dieser Aufgabe betrauen und mit den Spitälern, die an der Weiter- und Fortbildung mitwirken, vernetzt sind.

\section{Ist das Royal College ohne Konkurrenz?}

Schaut man in die Liste der Weiterbildungstitel, wird man stutzig: Es fehlt die Hausarztmedizin (Family Medicine) bei den Spezialfächern. Dieses Manko hat zu einer «Konkurrenz» geführt, dem College of Family Medicine. Dieses College, dessen Hauptquartier bei Toronto liegt, regelt den Weg zum Facharzttitel «family medicine» mit einem Weiterbildungsprogramm von zwei Jahren. In den staatlichen Spitälern sind die Programmverantwortlichen an den Fakultäten sowohl für das Royal College als auch das College of Family Medicine zuständig. Auf meine Fragen am Royal College in Ottowa stört man sich nicht an diesem Nebeneinander. Diese «kleine» Konkurrenz scheint die Grundfesten des Royal College nicht erschüttert zu haben und ist eher ein Addendum als eine standespolitische Bedrohung.

\section{Wermutstropfen im Weiterbildungs- wesen in Kanada}

Es besteht im kanadischen Modell eine Trennung zwischen der ärztlichen Interessenvertretung (Ärztegesellschaften der Teilstaaten) und der Weiterbildung. Bei den Weiterbildungsbelangen sind politische Steuerung (Anzahl Weiterbildungsplätze) und inhaltliche Ausgestaltung (Royal College) getrennt. Hört man von der Identifikation der Spezialisten mit «ihrem» College wirkt das Modell äusserst attraktiv. Doch auch hier fehlen die Schattenseiten nicht: Das Festlegen der Zahl der benötigten Fachärzte und somit der Weiterbildungsstellen, die bewilligt werden, hat seine Tücken. Es resultieren Engpässe in der spezialärztlichen Versorgung, die im Gegensatz zu früheren Jahren auch von der kanadischen Bevölkerung nicht mehr verstanden und nicht mehr stumm getragen werden. So mussten die Patienten in einzelnen Bereichen in die benachbarten USA zu Therapien geschickt werden, um Versorgungslücken auszuweichen. Der Staat musste für die Kosten aufkommen, bis die Versorgung mit erheblich finanziellem Aufwand wieder sichergestellt werden konnte.

Daneben sind immer mehr Ärzte im administrativ rigiden Gesundheitssystem unzufrieden und riskieren den Sprung in die Privatwirtschaft, ohne dass Versicherungen für die Kosten auf- 
kommen. Pressemitteilungen gemäss (z. B. in der Orthopädie) haben derart private Kliniken trotzdem Zulauf und schreiben mit Privateinnahmen schwarze Zahlen.

\section{Die anstehende Grundsatzdiskussion über das Facharztwesen}

Die Kosten des Gesundheitswesens und die Fehlleistungen der Planwirtschaft erfordern auch in Kanada eine Grundsatzdiskussion.

Viele Probleme sind eng mit den Belangen der Aus-, Weiter- und Fortbildung verknüpft. Am Sitz des Royal College konnte ich allerdings über den Stand einer solchen Diskussion nur wenig in Erfahrung bringen. Die fast revolutionären, drängenden Fragen, die Olli Miettinen [3] von McGill in Montreal bezüglich der Dauer und der Breite der Weiterbildung stellt, scheinen vor den Klostermauern haltzumachen. Die knappen Finanzen, die demographischen Veränderungen der Bevölkerung und die steigenden Ansprüche müssen zu kürzeren Aus- und Weiterbildungszeiten und zur gezielten Spezialisierung führen. Sowohl im kanadischen wie in unserem System der Weiterbildung muss die Bereitschaft erwachen, traditionelle Wege zu verlassen.

Dazu muss unsere Standesorganisation die Weiterbildung von den standespolitischen Aktivitäten «entkoppeln» (das können wir von Kanada lernen) und sich auch interprofessionellen Ansätzen der Gesundheitsversorgung öffnen (das wird auch hinter den Klostermauern von Ottawa zur Diskussion stehen müssen). Die lebenslange «Versorgung» der Ärztinnen und Ärzte mit Wissen muss das Kredo und der permanente Aktionsplan der Weiterbildungsorganisation werden.

Dass dabei die medizinischen Fakultäten eine wichtige Rolle einnehmen können (und sollen), ist (u.a. mit Blick auf Kanada) offensichtlich.
Der Bund, will er über die Aus-, Weiter- und Fortbildungsqualität der Ärzte die Wohlfahrt der Bevölkerung verbessern, kann auf die Expertise und die «Freiwilligenarbeit» unserer Weiterbildungsstätten und der Fachgesellschaften nicht verzichten. Alternativen lassen sich nicht aus dem Boden stampfen. Die europäische Freizügigkeit der Berufsausübung war Anlass, inhaltlich und formal im bestehenden System «über die Bücher zu gehen». Damit sind einige wunde Stellen aufgedeckt, aber noch keine Schritte zu grundsätzlich Neuem getan.

Die Grundsatzdiskussion über die Pfeiler der fachärztlichen Versorgung, über die Kosten der Weiterbildung und über das notwendige Mass an Wissen in der Grundversorgung muss zum Anliegen von Staat und Berufsorganisation werden. Die Fakultäten müssen vermehrt in diese Diskussion eingeschlossen sein und die Umsetzung gestalten.

Ein kanadischer Parlamentsbeschluss war es, der dem standespolitisch unabhängigen Royal College einen Auftrag zur Regelung der Weiterbildung gab. Wenn die FMH und ihre Fachgesellschaften die Weiterbildungsqualität von tariflichen Interessen trennen können, wird der politische Wille auch in der Schweiz vorhanden sein, die Verantwortung bei der Ärzteschaft zu belassen. Die Umgestaltung der Weiterbildung in Richtung Optimierung des Preis-Leistungs-Verhältnisses wird auch ohne Konkurrenzsystem möglich sein.

\section{Literatur}

1 FMPG: Bundesgesetz betreffend die Freizügigkeit des Medizinalpersonals in der Schweizerischen Eidgenossenschaft vom 19. Dezember 1877 (Stand am 7. Mai 2002).

2 MedBG: Bundesgesetz (Entwurf) über die universitären Medizinalberufe (Medizinalberufegesetz).

3 Miettinen OS. Medical curriculum and licensing: still in need of radical revision. Lancet 1992;340: 956-7. 